\title{
New tools could improve Alzheimer disease diagnosis from structural MRI
}

\begin{abstract}
ce
The model

could accu-

rately distin-

guish between

individuals

with $A D$ and

those with non-

neurodegenera-

tive conditions

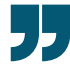

כ

sMRI images and measure atrophy.

In the first study, Hanne Struyfs

and colleagues developed a new

tool to perform this segmentation.

The tool, named icobrain $\mathbf{d m}$,

divides the sMRI image into

white matter, grey matter and cerebrospinal fluid then identifies the hippocampi and sub-regions of cortical grey matter.

The researchers applied icobrain dm to data from a cohort of healthy individuals and compared its performance with that of the existing image segmentation tool FreeSurfer as well as manual segmentation by experts. When compared with manual segmentation, both automated tools
\end{abstract}

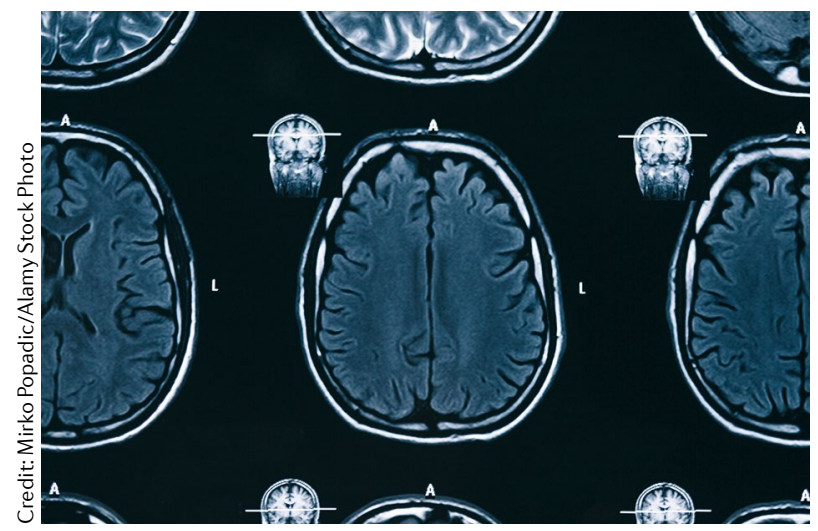

underestimated the volume of several brain regions; however, icobrain $\mathbf{~ d m}$ was more accurate than FreeSurfer.

Struyfs and colleagues then applied icobrain dm and FreeSurfer to data from a cohort comprising 46 individuals with $\mathrm{AD}$ and 23 controls and tested the ability of the tools to predict diagnosis on the basis of the volume of individual brain regions. Both tools were highly accurate, but the most accurate predictor was temporal lobe volume measured by icobrain $\mathbf{~ d m}$.

"We conclude that due to its low measurement error, icobrain $\mathbf{d m}$ could be of added value to the clinical diagnostic practice of AD patients," say the researchers in the paper.

"In future studies the performance of the measures to diagnose (very) early stages of $\mathrm{AD}$, as well as to distinguish between different dementia illnesses, should be further investigated."

In the second study, Lauren Koenig and colleagues used FreeSurfer to segment sMRI images, but designed a model that combined volumetric measurements from multiple brain regions to predict AD diagnosis. The researchers analysed publicly available sMRI data from a cohort of individuals with symptomatic $\mathrm{AD}$ and healthy controls and tested the ability of different combinations of volumetric measures to distinguish between the two groups of participants. The resulting model, named SARA, used data on brain regions including the hippocampus, inferior lateral ventricle, amygdala, and entorhinal and inferior parietal cortices to predict the diagnosis of individual participants.

SARA was tested on data from a separate cohort comprising individuals with $\mathrm{AD}$ and controls, and the model predicted diagnosis with a receiver operating characteristic (ROC) area under the curve (AUC) value of 0.961 . The closer the ROC AUC value is to 1 , the more accurate the diagnostic test. Hippocampal volume alone predicted diagnosis with a lower ROC AUC value than SARA, although the difference was not statistically significant. The researchers also adjusted SARA to take into account the trajectory of healthy age-related brain atrophy; however, this adjustment did not improve the diagnostic accuracy of the model.

Koenig and colleagues then tested the performance of SARA on data from a clinical cohort. The model could accurately distinguish between individuals with $\mathrm{AD}$ and those with non-neurodegenerative conditions, such as sleep disorders, and was also able to distinguish between $\mathrm{AD}$ and frontotemporal dementia. However, the researchers point out that this clinical cohort was too small for robust statistical analysis.

"SARA may be useful as a first step for selecting symptomatic $\mathrm{AD}$ participants for entrance into clinical trials or as an adjunct to the diagnostic algorithm when a clinical differential diagnosis includes $\mathrm{AD}$ versus frontotemporal dementia or non-neurodegenerative conditions," conclude the researchers in the paper.

Sarah Lemprière

ORIGINAL ARTICLES Struyfs, H. et al. Automated MRI volumetry as a diagnostic tool for Alzheimer's disease: validation of icobrain $\mathrm{dm}$. Neuroimage Clin. 26, 102243 (2020)| Koenig, L. N. et al. Select atrophied regions in Alzheimer disease (SARA): an improved volumetric model for identifying Alzheimer disease dementia. Neuroimage Clin. https://doi.org/10.1016/j.nicl. $2020.102248(2020)$ 